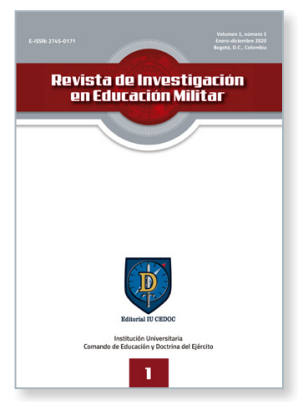

Revista de Investigación en Educación Militar

Volumen 1, número 1, enero-diciembre 2020, pp. 11-25

Bogotá, D. C., Colombia

E-ISSN: 2745-0171

https://doi.org/10.47961/27450171.3

\title{
Las ciencias militares en Colombia y el saber Doctrina Militar, alineación fundamental para la efectividad de las instituciones militares
}

\section{Edgar Rodríguez-Sánchez}

https://orcid.org/0000-0002-7622-7442

edgar.rodriguezsa@buzonejercito.mil.co

Comando de Educación y Doctrina del Ejército, Bogotá, D. C., Colombia

Citación: Rodríguez-Sánchez, E. A. (2020). Las ciencias militares en Colombia y el saber Doctrina Militar, alineación fundamental para la efectividad de las instituciones militares. Revista de Investigación en Educación Militar, 1(1), 11-25. DOI: https://doi.org/10.47961/27450171.3

Publicado en línea: $1 .^{\circ}$ de diciembre de 2020

Los artículos publicados por la Revista de Investigación en Educación Militar son de acceso abierto bajo una licencia Creative Commons: Atribución - No Comercial - Sin Derivados.

Para enviar un artículo:

https://revistascedoc.com/index.php/riem/about/submissions

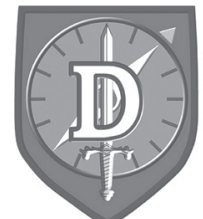

Editorial IU CEDOC 


\title{
Las ciencias militares en Colombia y el saber Doctrina Militar, alineación fundamental para la efectividad de las instituciones militares
}

\author{
The military sciences in Colombia and the Military Doctrine \\ knowledge, fundamental alignment for the effectiveness \\ of the military institutions
}

Edgar Alberto Rodríguez-Sánchez

Comando de Educación y Doctrina del Ejército, Bogotá, D. C., Colombia

\section{Resumen}

En Colombia, las ciencias militares reúnen varios saberes que, en conjunto, preparan a hombres y mujeres para desarrollar operaciones militares en los niveles de la guerra táctico, operacional y estratégico en los más de 160 programas académicos existentes, entre carreras técnicas, tecnológicas, de pregrado y de posgrado. Este artículo estudia la relación entre el saber Doctrina Militar y las ciencias militares a través de una metodología de cierre de brecha, mediante la cual se realizó un diagnóstico profundo de todas las mallas curriculares de los programas, incluyendo los syllabus, lo cual arrojó información sobre los obstáculos que impiden cumplir el propósito de la institución militar. Como conclusión se propone la ejecución de un plan que incluye soluciones tanto a la fundamentación de las ciencias militares como ciencias, como también a la aceleración del proceso de la producción de doctrina.

Palabras clave: capacitación; Colombia; doctrina; educación militar; formación; investigación.

\section{Abstract}

In Colombia, military sciences bring together various knowledge that, jointly, prepare men and women to develop military operations at the levels of tactical, operational and strategic warfare in more than 160 existing academic programs, including technical and technological careers, undergraduate and graduate studies. This article examines the relationship between Military Doctrine knowledge and military sciences through a gap-closing methodology, through which an in-depth diagnosis of all the curricular networks of the programs, including the syllabus, was made, which yielded information on the obstacles that prevent the fulfillment of the purpose of the military institution. As a conclusion, the execution of a plan is proposed and includes solutions both to the foundation of the military sciences as sciences, as well as to the acceleration of the process of doctrine production.

Keywords: Colombia; doctrine; investigation; military education; training

Artículo de investigación científica y tecnológica

Recibido: 9 de mayo de 2020

Aceptado: 16 de agosto de 2020

Contacto: Edgar Alberto Rodríguez-Sánchez $\bigotimes$ edgar.rodriguezsa@buzonejercito.mil.co 


\section{Introducción}

En los más de 160 programas de formación, capacitación, entrenamiento y reentrenamiento que tienen las ciencias militares en el Ejército colombiano, tanto en educación militar como en educación superior, se encuentran centenas de saberes y entre ellos está la Doctrina Militar como guía para el desarrollo de las operaciones militares. Actualmente, el Ejército de Colombia se encuentra en la actualización y jerarquización de la doctrina anterior a las Operaciones Terrestres Unificadas, con lo cual se pretende que la institución militar, en sus diferentes niveles organizacionales, realice el proceso de operaciones de acuerdo con su respectivo nivel de conducción de la guerra. En este sentido, y teniendo en cuenta el Proyecto Educativo de las Fuerzas Armadas (PEFA) (Ministerio de Defensa Nacional, 2014), el cual se encuentra en transición hacia la Política Educativa de la Fuerza Pública (PEFUP) (Ministerio de Defensa Nacional, 2019), este trabajo partió de la siguiente pregunta: ¿El programa de ciencias militares del Ejército colombiano está alineado a la doctrina de las Operaciones Terrestres Unificadas? De acuerdo con esta pregunta, se estableció como objetivo de la investigación analizar el saber de Doctrina Militar respecto a las ciencias militares, con el fin de observar el ciclo propedéutico en el proceso de formación y capacitación, así como en los procesos de instrucción y entrenamiento de acuerdo con su misión constitucional.

La investigación formó parte de uno de los equipos de trabajo pertenecientes a la Institución Universitaria Comando de Educación y Doctrina del Ejército Colombiano (IUCEDOC), donde se realizó un estudio similar con otros saberes, como: (1) Investigación, comunicación y segunda lengua; (2) Psicología, medio ambiente, preservación y sanidad; (3) Normatividad militar, Derechos Humanos, ética y liderazgo; (4) Administración y gestión logística; (5) Preparación táctica, inteligencia, historia, orientación institucional y doctrina; (6) Preparación técnica, orientación militar, cultura física y orden cerrado; (7) Directiva, y (8) Ciencia y tecnología.

\section{Marco teórico}

Esta investigación se enmarca en la disciplina de las ciencias militares y la categoría de la doctrina militar. En el transcurso de la historia se ha cuestionado sobre el conocimiento y, en consecuencia, los diferentes teóricos han tenido una concepción diferente de este. El saber, conocer, pensar y la veracidad de estos conceptos se ha debatido hasta la actualidad, pues no cesa la voluntad de entender el mundo y sus complejidades a través de una serie de elementos y procesos que dan cabida y, sobre todo, posibilitan el conocimiento indagado a través de la epistemología (Najmanovich, 2008).

En tal sentido, y si la cuestión central radica en el conocimiento mismo, son diversos los enfoques y escuelas que desde la epistemología se han encargado de dilucidarlo a través de múltiples debates sin que hayan llegado a un consenso unívoco. Sin embargo, ¿cómo llevar a cabo la justificación de este conocimiento?, ¿qué nos permite esta justificación? Así, la 
justificación tiene que permitirnos afirmar con cierta seguridad cuándo sabemos y cuándo no sabemos. Hablar de epistemología es hablar sobre el conocimiento, su estudio, su veracidad, su justificación. Según el filósofo y epistemólogo británico Jonathan Dancy, la epistemología:

es el estudio del conocimiento y de la justificación de la creencia. Sus cuestiones centrales radican en las preguntas: "¿Qué creencias están justificadas y cuáles no?", "Si hay algo que podemos conocer, ¿qué es?", "¿Cuál es la relación entre conocer y tener una creencia verdadera?", "¿Cuál es la relación entre ver y conocer?". (Dancy, 1993, p. 15)

El Consejo Nacional de Acreditación (CNA) de la República de Colombia conceptualiza el conocimiento como la agrupación que se hace de los programas académicos, teniendo en cuenta cierta afinidad en los contenidos, en los campos específicos del conocimiento y en los campos de acción de la educación superior, cuyos propósitos de formación conduzcan a la investigación o al desempeño de ocupaciones, profesiones y disciplinas. De acuerdo con el CNA, las áreas de conocimiento son ocho: (1) Agronomía, veterinaria y afines; (2) Bellas artes; (3) Ciencias de la educación; (4) Ciencias de la salud; (5) Ciencias sociales y humanas; (6) Economía, administración, contaduría y afines; (7) Ingeniería, arquitectura, urbanismo y afines, y (8) Matemáticas y ciencias naturales (Consejo Nacional de Acreditación, 2020). Entre los 55 núcleos básicos del conocimiento se encuentra el núcleo básico "Formación relacionada con el campo militar y policial", lo cual permite afirmar que las ciencias militares son parte de la gran área del conocimiento de las ciencias sociales y humanas.

Siguiendo la línea de la epistemología de las ciencias sociales y humanas, las ciencias militares se definen como el conjunto de disciplinas que se encargan de estudiar, de forma sistémica, los procesos militares, filosóficos, políticos, económicos y psicosociales que son producto del ser humano y su relación con la guerra (Ryabchuk, 2001). En un sentido más funcional, la doctrina de las Operaciones Terrestres Unificadas "Damasco" también define que las ciencias militares

\begin{abstract}
tratan de la conducción de la guerra. Si bien la naturaleza de la guerra es constante, esta cambia permanentemente. La guerra se ve afectada por los cambios en el Derecho Internacional, la tecnología, las estructuras de organización, los conceptos operacionales, la opinión pública, las políticas nacionales y muchos otros factores. La conducción de la guerra puede tener similitudes en el tiempo, pero también tendrá grandes variaciones. (Ejército Nacional de Colombia, 2016, p. 24).
\end{abstract}

De acuerdo con la doctrina militar, "los profesionales en Ciencias Militares del Ejército emplean la doctrina en dos contextos: estudio y reflexión, así como conducción (planeamiento, preparación, ejecución y evaluación) de operaciones" (Ejército Nacional de Colombia, 2016, p. 3). El enfoque, el método, la metodología, las técnicas y los instrumentos son los que permiten justificar el conocimiento de las ciencias militares. 
Dentro de estas ciencias militares se encuentra la doctrina militar, la cual debe ser tanto teórica como práctica. La doctrina no es un catálogo de respuestas para la solución de problemas específicos, sino que por el contrario es un "compendio de fundamentos, tácticas, técnicas y procedimientos para el pensar sobre problemas militares, qué operaciones son las más complejas y qué acciones las resuelven mejor" (Ejército Nacional de Colombia, 2016, p. 3). También la doctrina militar se define detalladamente como los

principios fundamentales con sus correspondientes tácticas, técnicas, procedimientos, términos y símbolos empleados para la conducción de las operaciones y mediante los cuales el Ejército de combate y los elementos del Ejército generador, que apoyan directamente las operaciones, guían sus acciones en cumplimiento de los objetivos nacionales. (Ejército Nacional de Colombia, 2016, p. 55)

La doctrina militar, a través del manual de técnicas MTE 5-0.1, de arte y diseño operacional, plantea la manera en que se debe desarrollar una operación militar en los niveles estratégicos. Además, al enmarcar los problemas, emplea el análisis sistémico basado en las variables operacionales -lo político, económico, militar, social, información, infraestructura, entorno físico y tiempo (PEMSITIM) (Ejército Nacional de Colombia, 2019, pp. 3-17) - y las de la misión — como misión, enemigo, terreno y clima, tropas y apoyo disponible, tiempo disponible y consideraciones civiles (METT-TC) (Ejército Nacional de Colombia, 2019, pp. 5-2)—, las cuales facilitan a los comandantes de unidades militares y al estado mayor enmarcar el ambiente operacional y les proporcionan un marco de referencia común para que interactúen y colaboren con los asociados de la acción unificada, entendiendo el ambiente operacional desde todas las perspectivas (las ciencias militares), además del puro de la ciencia militar.

El saber Doctrina Militar debe estar estandarizado dentro de los programas, mallas académicas, syllabus, competencias y contenidos, de acuerdo con los contenidos curriculares establecidos (Ministerio de Defensa Nacional, 2010). Asimismo, debe tener en cuenta la transversalización y la propedéutica a lo largo de la carrera militar, sin perder el horizonte de los objetivos estratégicos del Ejército Nacional, los cuales se encuentran alineados con las áreas del sector defensa de Colombia: (1) la defensa nacional; (2) la seguridad pública; (3) la contribución al desarrollo del país; (4) la protección del medio ambiente y los recursos naturales; (5) la cooperación internacional, y (6) la gestión del riesgo y los desastres (Ciro \& Correa, 2014).

\section{Método y metodología}

La investigación empleó un método inductivo y cualitativo. Específicamente, como metodología usó el cierre de brecha, que se basa en el trabajo de Alfred Chandler (2003), en el cual la 
determinación de las metas y los objetivos básicos de largo plazo en una institución, junto con la adopción de cursos de acción y la distribución de los recursos necesarios permiten lograr los propósitos que esta se ha planteado (Chandler, 2003). En este sentido, la metodología que se empleó para integrar y alinear el plan estratégico del Ejército colombiano con el propósito tuvo seis pasos: (1) análisis del contenido semántico del propósito; (2) establecer los criterios de logro (o condiciones) que permitan conocer o saber si se ha cumplido el propósito; (3) establecer la situación actual de la institución frente a cada uno de los criterios de logro definidos en el paso número 2; (4) identificar los problemas que impiden cerrar la brecha entre la situación deseada y la situación actual; (5) definir el impacto entre objetivos e iniciativas estratégicas, y (6) la ejecución y puesta en marcha de las acciones o iniciativas.

Las técnicas de recolección de información se aplicaron a la búsqueda de fuentes primarias y secundarias para construir el estado del arte y de los fundamentos teóricos. De la misma forma, al ser una investigación cualitativa, los datos y contenidos identificados en las consultas de fuentes primarias y secundarias se sistematizaron en una base de datos, con especial énfasis en los procesos y procedimientos doctrinales. Finalmente, la metodología cualitativa se complementó con entrevistas esporádicas y, por último, con la estrategia de "bola de nieve", con el fin de recopilar más información de acuerdo con las lecturas compartidas y cruzadas que se realizaron en la búsqueda documental.

\section{Resultados}

El Comando de Educación y Doctrina del Ejército Nacional de Colombia (CEDOC) generó un sistema de educación con base en el Proyecto Educativo de la Fuerza Pública (PEFA) y que se ha alimentado con los objetivos del Estado en cuanto a la seguridad y defensa. En este sentido, la institución militar colombiana proyecta estandarizar las mallas curriculares del saber Doctrina Militar con el fin de incrementar la efectividad del Ejército en el cumplimiento de su misión constitucional, para lo cual tiene como referencia el Proyecto Educativo Institucional (PEI) (Ejército Nacional de Colombia, 2015), el Comité Estratégico de Diseño del Ejército del Futuro (CEDEF) y el Plan MINERVA

Al revisar las mallas curriculares y los programas que existen en el Ejército colombiano se pudo observar que varias disciplinas se encuentran inmersas en el ámbito militar, como la historia, la sociología, la antropología, la ciencia política, la psicología, el derecho, la salud operacional, la administración y la educación, las cuales se encuentran muy relacionadas con las ciencias militares. Asimismo, el análisis de la situación actual de las ciencias militares en el Ejército de Colombia evidenció que no había una epistemología de las ciencias militares en las diferentes mallas curriculares de formación y capacitación, ni se conocía el proceso de operaciones como metodología científica para desarrollar las campañas, los planes, el orden de operaciones y las misiones tácticas, así como tampoco había 
profesionales en ciencias militares con perfil suficiente para realizar diagnósticos sobre ciencias militares y doctrina que revisaran los programas.

\section{Paso 1. Análisis del contenido "semántico" del propósito}

El análisis de contenido "semántico" del propósito se hizo con el ciclo-epistemológico, aplicado en la formación del conocimiento. Es decir, el propósito se dividió en las ideas subyacentes y luego se conceptualizó cada idea con el fin de hacer las aclaraciones que fueron necesarias y pertinentes para poder minimizar las imprecisiones y ambigüedades que estaban inmersas en el enunciado del propósito.

Las ciencias militares y la doctrina militar ya fueron definidas en el marco teórico de esta investigación y, por lo tanto, el análisis se relaciona con el propósito de fortalecer las ciencias militares mediante la evaluación y la organización por ciclos propedéuticos en los procesos de formación, capacitación, instrucción, entrenamiento y reentrenamiento aplicando permanentemente la doctrina militar Damasco.

Como las ciencias se fundamentan en el método científico para confirmar hipótesis, el programa de Ciencias Militares contempla la investigación en las líneas de Educación y formación militar, Instrucción y entrenamiento militar, y Gestión y educación ambiental (ESMIC, 2020). Por lo tanto, falta contemplar el sentido holístico de la interdisciplinariedad, especialmente la ciencia militar pura sobre la conducción de la guerra.

En cuanto a la doctrina militar, el proyecto educativo del programa Profesional en Ciencias Militares organiza este saber en el área Profesional Específica (ESMIC, 2020). Con base en esta información se puede inferir que la alineación de las ciencias militares solo se hace a través de esta área, de manera que deja por fuera las Áreas Básicas, Investigativa y Sociohumanística, fundamentales para la formación integral del profesional en Ciencias Militares. De acuerdo con el Proyecto ESMIC, el saber Doctrina Militar proporciona identidad acerca de

quiénes son los militares, en qué creen, para qué existen, a quién sirven, qué valoran, qué hacen, cómo lo hacen, de dónde vienen, hacia dónde van y de dónde partirán, la que se construye y define en dependencia de la sistematización de las experiencias prácticas; de la interpretación de los contextos y entornos desde las alianzas estratégicas en el sistema internacional; la prospectiva nacional; la tecnología más avanzada, no comprendida como instrumentos y aparatos técnicos solamente, sino metodologías, organización, gestión, administración, entre otros conocimientos de las ciencias militares y de su investigación científica. (ESMIC, 2020, p. 7)

La Escuela Militar de Cadetes "General José María Córdova" tiene el programa de Ciencias Militares para formar comandantes de pelotón en la dimensión táctica, pero no contempla la dimensión operacional ni la dimensión estratégica, ya que este nivel de formación corresponde a otras escuelas, como las del Centro de Educación Militar y la Escuela Superior 
de Guerra. En el programa del pregrado de Ciencias Militares, no se conceptualiza la etimología de las ciencias militares, pero se hace una aproximación al concepto, hacia su objetivo, el cual es planear, investigar, dirigir, administrar, conducir y evaluar las situaciones que la profesión del ser militar lo demanden (ESMIC, 2020). El programa desarrolla este objetivo a través de las áreas del conocimiento humanístico, social, tecnológico, metodológico y de otras ciencias básicas dentro de las Áreas Básicas, Profesional, Investigativa y Sociohumanística. Por lo tanto, se puede afirmar que existe una interdisciplinariedad en las ciencias militares y, en consecuencia, una alienación al concepto de Ryabchuck (2001).

En cuanto a la Escuela Militar de Suboficiales "Sargento Inocencio Chincá", se encontró que en sus programas de Tecnología en Ciencias Militares no se conceptualizan las ciencias militares, pero coinciden en que la investigación para los programas de formación de la IU-CEDOC tiene como objetivo generar información y conocimiento sobre diversos procesos naturales, sociales, económicos, políticos, culturales, jurídicos y tecnológicos para contribuir a la solución de problemáticas que se presenten en la realidad nacional. Asimismo, se divide en las áreas de Formación Básica, Sociohumanista, Investigación, Formación Profesional General y Formación de Profesional Específica (Ministerio de Defensa Nacional, 2010).

Teniendo en cuenta el anterior diagnóstico, se pudo determinar que existe una directiva estructural para la gestión de la historia militar (Directiva Estructural del Comando del Ejército 00090 del 2018) y un proceso de certificación en el Sistema Integrado de Gestión (SIG) denominado "Socio humanística y cultura militar", los cuales guían a las unidades militares en el desarrollo de las investigaciones en ciencias sociales y humanas aplicadas al ámbito militar.

En cuanto a la relación de las ciencias militares con la doctrina militar, se pudo establecer que los manuales que contienen información que no esté relacionada directamente con la conducción y ejecución de las operaciones militares se clasifican como otras publicaciones. En estas publicaciones se encuentra el Manual de educación militar (MEM), el cual contiene conceptos y procedimientos relacionados con la instrucción, la educación y el entrenamiento del personal. Aun cuando esta publicación no es doctrina, sí se basa en ella — por ejemplo, puede contener información sobre cómo se entrena para un evento específico o sobre algún equipo o sistema de armas - No obstante, no describe el mantenimiento y empleo de material o equipo, ni los fundamentos, las tácticas, las técnicas, los procedimientos o los términos y símbolos — de ser necesario, hace la respectiva referencia al manual proponente- - Como las publicaciones doctrinales, estos manuales de educación militar deben cumplir con los mismos procesos de aprobación y emplear términos y símbolos contenidos en el MFRE 1-02 (Ejército Nacional de Colombia, 2017b).

A partir de este diagnóstico preliminar, se empleó una matriz adicional para analizar el saber Doctrina Militar dentro de los diferentes niveles de formación y capacitación, como también en los diferentes niveles de la guerra (tabla 1). 
Tabla 1. Matriz de análisis del saber Doctrina Militar respecto a las ciencias militares

\begin{tabular}{cccc}
\hline Nivel de la guerra & Educación militar* & $\begin{array}{c}\text { Nivel de educación } \\
\text { Ciencias Militares }\end{array}$ & $\begin{array}{c}\text { Lineamientos } \\
\text { curriculares SEFA }\end{array}$ \\
\hline $\begin{array}{c}\text { Táctico } \\
\text { (Hasta nivel Compañía) }\end{array}$ & $\begin{array}{c}\text { Formación } \\
\text { (EMSUB/ESMIC/ESPRO) }\end{array}$ & $\begin{array}{c}\text { Técnica } \\
\text { (Soldados) }\end{array}$ & Básica \\
\hline $\begin{array}{c}\text { Operacional } \\
\text { (Hasta nivel Unidad } \\
\text { Operativa Mayor) }\end{array}$ & $\begin{array}{c}\text { Capacitación } \\
\text { (CEMIL/CEMAl/CEHEJ/ } \\
\text { CEDOE) }\end{array}$ & $\begin{array}{c}\text { Tecnológica } \\
\text { Suboficiales) }\end{array}$ & Sociohumanística \\
\hline $\begin{array}{c}\text { Estratégico } \\
\text { (Hasta Fuerzas Militares y } \\
\text { Ministerio de Defensa) }\end{array}$ & $\begin{array}{c}\text { Instrucción } \\
\text { (CENAE/BRIER) }\end{array}$ & $\begin{array}{c}\text { Pregrado } \\
\text { (Oficiales) }\end{array}$ & Profesional \\
\hline & $\begin{array}{c}\text { Entrenamiento } \\
\text { (CENAE/BRIER) }\end{array}$ & $\begin{array}{c}\text { Especialización } \\
\text { (Abierta) }\end{array}$ & Investigativa \\
\hline & $\begin{array}{c}\text { Maestría } \\
\text { (Abierta) }\end{array}$ \\
\hline & $\begin{array}{c}\text { Doctorado } \\
\text { (Abierta y a futuro) }\end{array}$ & \\
\hline
\end{tabular}

* Unidades de la IUCEDoc: Escuela Militar de Suboficiales (EMSUB), Escuela Militar de Cadetes (ESMIC), Escuela de Soldados Profesionales (ESPRO), Centro de Educación Militar (CEMIL); Centro de Misiones Internacionales y Acción Integral (CEMAI), Centro de Estudios Históricos del Ejército (CEHEJ), Centro de Doctrina del Ejército Nacional (CEDOE), Centro Nacional de Entrenamiento (CENAE) y la Brigada de Instrucción, Entrenamiento y Reentrenamiento (BRIER).

Fuente: elaboración propia.

\section{Paso 2. Brecha estratégica}

El segundo paso consistió en establecer los criterios de logro (o condiciones) que permitan conocer o saber si se ha logrado el propósito. Es decir, ¿cómo saber si se ha podido lograr el propósito?, ¿qué factores o condiciones deberían cumplirse para poder decir que se ha alcanzado el propósito? Tales criterios de logro no son otra cosa que "los factores" o "condiciones" que deben estar presentes o cumplirse para poder saber si se ha cumplido o no el objetivo propuesto.

El paso número dos de la metodología tuvo como objetivo describir los criterios de logro, definidos como evaluaciones implícitas que se utilizan para saber si se está cumpliendo con el objetivo o no. Dichos criterios de logro debían ser definidos por tema, no en general. Para esta actividad, y teniendo en cuenta el paso número 1, se verificaron las mallas curriculares, los syllabus, las competencias y las temáticas de los más de 165 programas con los que cuenta la IUCEDOC, con el fin de establecer los componentes necesarios para cumplir el propósito de fortalecer las ciencias militares con el saber Doctrina Militar.

La palabra doctrina viene de una profesionalización de hace más de doscientos años y se define como los principios fundamentales con sus correspondientes tácticas, técnicas, pro- 
cedimientos, términos y símbolos empleados para la conducción de las operaciones mediante las cuales el Ejército de combate y los elementos del Ejército generador de fuerza que apoyan directamente a las operaciones guían sus acciones en cumplimiento de los objetivos nacionales. Este concepto se adopta desde la independencia de los españoles en 1810 y durante el siglo XIX el Ejército colombiano tuvo una doctrina francesa napoleónica, que cambió hasta el siglo XX, cuando viró hacia la doctrina prusiana en 1907, luego la doctrina suiza, la doctrina alemana, la doctrina francesa y a partir de 1942 se adoptó la doctrina americana (Cardona, 2020). Desde ese momento y hasta el año 2016, la doctrina se ha dividido en dos: la doctrina convencional (o regular) y la doctrina interna (de contrainsurgencia o irregular). La doctrina regular (americana) tuvo una evolución hasta el presente, mientras que la otra se basó en el modelo desarrollista, pero se sumó a la doctrina francesa y americana, es decir, la doctrina ecléctica, que es la que maneja el orden público interno y se basa en la acción integral (Cardona, 2020).

Por otro lado, la doctrina regular empezó con la defensa activa de la amenaza de los misiles en la Guerra Fría, y en 1984 se empezó a aplicar la doctrina de la batalla aeroterrestre, es decir, cuando se empiezan a utilizar todos los campos activos del sistema de combate. Pero cuando llegó la Guerra del Golfo Pérsico en 1991, la doctrina evolucionó al full dimension o 'dimensión total', con el fin de abarcar todos los lugares donde podrían existir ataques (agua, tierra y aire). Con la Guerra de Irak se empezó a pensar en una doctrina mayor, la del espectro total, que abarcó también los dominios del espacio y el ciberespacio, dentro de todo el espectro de las operaciones tanto de guerra como las operaciones diferentes a la guerra (Cardona, 2020).

Entonces, con el fin de alinear todas las doctrinas existentes en el tiempo, el Ejército de Colombia desarrolló el proyecto Damasco para adoptar la doctrina de operaciones terrestres unificadas y jerarquizar los diferentes manuales. En este sentido, y teniendo en cuenta lo anterior, los criterios de logro definidos en este paso se propusieron cumplir: (1) Cuando el manual de Arte y diseño operacionaly Proceso de operaciones del Ejército incluyera los conceptos epistemológicos de las ciencias militares; (2) Cuando la misión o los ejercicios de planeamiento se desarrollaran bajo la Doctrina Damasco de acuerdo con los manuales fundamentales de referencia del Ejército Nacional; (3) Cuando se realizara investigación formativa y formal en doctrina militar; (4) Cuando la Doctrina Damasco se encontrara en las mallas curriculares de los diferentes programas del sistema de educación de acuerdo con el nivel propedéutico, y (5) Cuando la Doctrina Damasco con base en las capacidades y funciones de conducción de la guerra fuera entendida y aplicada por los integrantes de la institución militar.

\section{Paso 3. Alineación estratégica}

En este paso se estableció la situación actual de la institución frente a cada uno de los criterios de logro definidos en el paso número 2, la cual fue soportada con fuentes primarias. 
El proceso de jerarquización y actualización de la doctrina inició con el Plan MINERVA en el 2015, en el marco del proyecto Damasco, que se alineó con las Operaciones Terrestres Unificadas. Cinco años después de que el proceso inició, se han concretado 17 de 17 Manuales Fundamentales del Ejército (MFE); 17 de 17 Manuales Fundamentales de Referencia del Ejército (MFRE); 21 de 55 Manuales de Campaña del Ejército y 11 de 276 Manuales de Técnicas del Ejército. Adicionalmente, se han concretado 11 Reglamentos, 6 Manuales de Educación Militar y 6 Cartillas Doctrinales ${ }^{1}$.

De acuerdo con el Manual Fundamental del Ejército MFE 1-01, los manuales administrativos del Ejército (MAE), de Educación Militar del Ejército (MEM) y el Manual de Mantenimiento y Empleo de Material y Equipo del Ejército (MME) no son doctrina porque no abordan tácticas, técnicas ni procedimientos para el desarrollo de operaciones militares, pero apoyan a la doctrina como otras capacidades militares (Ejército Nacional de Colombia, 2016).

Los manuales MFE 5-0 de Proceso de operaciones y 5-0.1 de Arte y diseño operacional contemplan los métodos, la metodología, las técnicas y los instrumentos de las ciencias militares, incluyendo parte de su epistemología, pero dejan por fuera aspectos propios de las ciencias como el fundamento del conocimiento del ser en la guerra (la ciencia militar pura) (Ejército Nacional de Colombia, 2017a).

Asimismo, se encontró que debido a que el avance de la Doctrina Damasco está al 26 \%, los manuales que se emplean en el nivel táctico corresponden a la anterior doctrina y son usados como manuales de campaña y de técnicas.

No existe investigación formativa o formal sobre doctrina en el Ejército Nacional. Los programas de algunas escuelas no tenían contemplado la investigación, ni se observaban los bancos de proyectos que pudieran indicar si estaba proyectada la investigación en doctrina militar. La investigación de doctrina militar la estaba desarrollando el CEDOE con métodos y técnicas sin definir el método científico estándar.

Una vez fueron revisadas las mallas curriculares y los syllabus de los 153 cursos que se desarrollaban en el CEMIL, la malla curricular del pregrado de Ciencias Militares de la Escuela Militar de Cadetes, la malla curricular del pregrado en Tecnologías Militares de la Escuela Militar de Suboficiales, y las mallas de entrenamiento de soldados, reentrenamiento de tropas, especialistas y dragoneantes, se observó que todas cumplían con la alineación de tres de las cuatro áreas contempladas en el SEFA (Básica, Profesional, Sociohumanística e Investigativa), con excepción de la ESMIC y la EMSUB.

Las áreas Básicas, Profesional, Sociohumanística e Investigativa están alineadas con las áreas de Preparación Táctica, Técnica, Sociohumanística y Física, de manera que la doctrina se transversaliza en todas las áreas, pero no es mencionada como un saber propio, con excep-

1 Diagnóstico realizado con el Centro de Doctrina del Ejército Nacional. 
ción de la ESMIC y la EMSUB. Aunque la doctrina está implícita en todas las áreas, no había un objetivo introductorio de doctrina en los diferentes saberes que correspondían a la ciencia militar pura (ofensivas, defensivas, etc.).

\section{Paso 4. Problemas y obstáculos}

En este paso se identificaron los problemas que impedían cerrar la brecha entre la situación deseada y la situación actual, al igual que las soluciones (iniciativas estratégicas) que permiten superarlos; es decir, ilustra la identificación de un problema y su posible solución. Haciendo uso de "la imaginación y la creatividad", se exploraron las posibilidades de mejora o cambios radicales que posiblemente permiten superar los problemas identificados. En este sentido, se debe mencionar que se encontraron varios problemas en este cierre de brecha.

En primera medida, se estableció que la institución militar no cuenta con personal suficiente para acelerar el proceso de la Doctrina Damasco, ya sea porque algunas de las personas que realizan los manuales no tienen el perfil de escritores — no cuentan con la disciplina o la cultura de la rigurosidad académica - o porque no hay un plan para acelerar el proceso en cuanto a la producción de los manuales. Tampoco se cuenta con la epistemología de las ciencias militares en las diferentes mallas curriculares de formación y capacitación.

Como segundo aspecto, se estableció que las mallas curriculares que tenían relación con las funciones de conducción de la guerra no formulaban una tarea introductoria a la doctrina ni su función dentro del planeamiento de las operaciones. Asimismo, de acuerdo con un sondeo entre varios integrantes del Ejército que participaron en la investigación, se encontró que no había conocimiento de los 66 manuales que había emitido el CEDOE respecto a la doctrina de Operaciones Terrestres Unificadas. Tampoco se conocía el proceso de operaciones como metodología científica para desarrollar las campañas, los planes, el orden de operaciones y las misiones tácticas, ni la doctrina militar se encontraba dentro de la base de proyectos de los diferentes grupos de investigación.

Como tercer aspecto, referente a la investigación, la doctrina militar no se encontró dentro de la base de proyectos de los diferentes grupos de investigación registrados ante el Ministerio de Ciencia, Tecnología e Innovación. Tampoco se encontraba la investigación sobre doctrina militar dentro de la agenda de proyectos o planes de producción en los pregrados y posgrados, como especializaciones y maestrías. Asimismo, había programas que no contenían el área Investigativa debido al corto tiempo de duración, ni había convocatorias ni estímulos para la investigación formativa o formal en doctrina militar.

Como cuarto aspecto, se identificó que no se había revisado detalladamente la propedéutica en doctrina militar ni su competencia en los diferentes niveles de la guerra, como tampoco se encontraron diagnósticos sobre ciencias militares y doctrina. 


\section{Discusión}

\section{Paso 5. Propuestas de soluciones}

En este paso se establecieron los impactos de las iniciativas. En este sentido, la propuesta de solución a los obstáculos encontrados podría ser la ejecución de un plan interno que incluyera:

- Propender hacia la formación de teóricos en epistemología o filosofía que puedan darle relevancia a las ciencias militares como ciencias y que puedan construir un manual de fundamentos epistemológicos de las ciencias militares, así como incluir estos fundamentos en los diferentes programas de formación y capacitación, alineándolos con el proceso de operaciones, el arte y el diseño operacional con sus respectivas metodologías.

- Incluir en los diferentes programas de formación y capacitación los fundamentos epistemológicos de las ciencias militares, como también la temática de introducción a la doctrina y su función dentro del planeamiento de las operaciones en los saberes que tengan relación con las funciones de conducción de la guerra. En cuanto a la difusión de la doctrina y su enseñanza, como primera medida se debe acelerar el proceso de producción de manuales mediante la asignación de los manuales técnicos a expertos o personal con perfil que se encuentren en otras unidades militares.

- En cuanto a la investigación en doctrina militar, se propone como primera medida incluir en la investigación formal y formativa la línea de investigación en doctrina militar, como también incrementar las convocatorias en ciencias sociales y humanas, verificando que los GrupLAC tengan incluida la doctrina militar en las agendas de proyectos. Como segunda medida, la solución incluye crear semilleros de investigación que se vinculen a los GrupLAC de la institución militar. En esta solución, los proyectos de doctrina militar deben incluir los investigadores docentes, junior, asociados y senior para aumentar la rigurosidad académica. En los programas que no tienen área de investigación, se debe fomentar la investigación en doctrina militar y, para aumentar la métrica de los GrupLAC, se debe asignar el personal que tenga posgrados y esté reconocido por Minciencias como tutores de los pregrados y posgrados en Ciencias Militares.

\section{Paso 6. Ejecución y puesta en marcha del plan estratégico}

Este paso es complejo de ejecutar debido a las condiciones propias de la institución militar, pues se debe tener presente el compromiso de sus integrantes en el desarrollo de las operaciones militares. La ejecución y puesta en marcha del plan se facilitarían con un modelo de gestión integral, donde se evalúe el cumplimiento de los objetivos (efectividad), el nivel de avance de las acciones o iniciativas estratégicas (eficacia) y finalmente el uso y manejo de recursos (eficiencia). El esquema de evaluación propuesto forma parte de un 
sistema de control de gestión, el cual debe incluir los procedimientos que rigen al modelo y un sistema de información que soporte el flujo y manejo de la información.

\section{Conclusiones}

Las ciencias militares tratan de la conducción de la guerra. En Colombia esta epistemología reúne varios saberes que, en conjunto, preparan a los hombres y mujeres en los niveles de educación técnica, tecnológica, de pregrado y posgrado para desarrollar operaciones militares en los niveles de la guerra táctico, operacional y estratégico. Dentro de estas ciencias, se encuentra el saber Doctrina Militar, que guía el desarrollo de las operaciones militares a través de pasos, técnicas y procedimientos.

El Ejército de Colombia, en cumplimiento de su misión constitucional, debe garantizar los derechos y libertades de los ciudadanos que habitan el territorio y para ello debe contar con hombres y mujeres preparados para tal fin. En este sentido, las ciencias militares, como programa para la formación y capacitación de los integrantes de la institución militar en los diferentes niveles de la guerra, se enmarcan dentro de las exigencias de tres ministerios colombianos para cumplir con el mandato constitucional: el de Defensa, el de Educación y el de Ciencia, Tecnología e Innovación.

Teniendo como base los objetivos estratégicos de la institución militar, la investigación propuso la siguiente pregunta de investigación: ¿El programa de Ciencias Militares del Ejército colombiano está alineado a la doctrina de las Operaciones Terrestres Unificadas"? Empleando la categoría de doctrina militar y su relación con las ciencias militares, a través de la metodología de cierre de brecha se encontró que la alineación no está realizada efectivamente, ya que el diagnóstico indica una serie de obstáculos que se deben solucionar para llegar a las condiciones óptimas.

La metodología permitió clarificar los conceptos de ciencias militares y doctrina, así como definir cinco criterios de logro para cumplir el propósito de la institución, el cual es fortalecer las Ciencias Militares con la doctrina militar. Los criterios de logro razonables para la institución militar incluían conceptos epistemológicos; planeamiento de operaciones de acuerdo con los Manuales Fundamentales de Referencia del Ejército; investigación formativa y formal en doctrina militar; adecuación de las mallas curriculares con nivel propedéutico en doctrina militar, y la aplicación de la doctrina militar por los integrantes de la institución militar.

Asimismo, se reconoce la importancia que tiene la unificación doctrinal en los niveles de formación profesional, tecnológico y técnico, pues genera fatores de aprendizaje reales y coherentes con su estructura básica. Esta unificación se debe hacer desde el concepto básico de doctrina, de tal manera que se desarrolle en la función de conducción de la guerra, así como en los aspectos relacionados con los diferentes procesos y teniendo en cuenta su función en el dominio táctico. 
En el ejercicio de investigación se estableció que existen más de 160 programas académicos entre carreras técnicas, tecnológicas, de pregrado y de posgrado. El diagnóstico profundo de todas las mallas curriculares de los programas, incluyendo los syllabus, arrojó información sobre distintas situaciones actuales que son relevantes, como también sobre obstáculos que impedían cumplir el propósito de la institución militar. Entre los resultados más significativos se encontró que la epistemología de las ciencias militares no está bien definida ni se encuentra en la doctrina militar. Asimismo, este diagnóstico también evidencia que no hay claridad sobre la ciencia, sus enfoques, marcos teóricos, métodos, metodologías, técnicas e instrumentos.

Otro dato relevante es que la actualización de la doctrina militar se encuentra en el $26 \%$. En este sentido, cinco años después de iniciado el proceso, se han concretado 17 de 17 Manuales Fundamentales del Ejército (MFE); 17 de 17 Manuales Fundamentales de Referencia del Ejército (MFRE); 21 de 55 Manuales de Campaña del Ejército y 11 de 276 Manuales de Técnicas del Ejército. Estos resultados muestran que la alineación de esta doctrina con las ciencias militares no está en el punto óptimo, de manera que es necesario proponer soluciones efectivas para alcanzar la condición deseada.

La investigación, además de responder a la pregunta de investigación, propone la ejecución de un plan que incluye soluciones tanto a la fundamentación de las ciencias militares como ciencias, como también a la aceleración del proceso de la producción de doctrina y su alineación con las mallas curriculares de los diferentes programas de formación y capacitación existentes en la institución militar.

La ejecución del modelo propuesto permite que la institución militar responda a las exigencias de los tres ministerios colombianos y pueda ser efectiva en el desarrollo de las operaciones militares. Además, plantea que los procesos de investigación se empleen como retroalimentación de la doctrina militar cuando se evalúan las mismas operaciones, con lo cual se cumpliría con el método científico que hace posible una ciencia.

\section{Agradecimientos}

El autor desea agradecer al Comando de Educación y Doctrina del Ejército por su apoyo en la realización de este artículo.

\section{Declaración de divulgación}

El autor declara que no existe ningún potencial conflicto de interés relacionado con el artículo. La presente investigación forma parte del proyecto de fortalecimiento de las ciencias militares del Comando de Educación y Doctrina del Ejército.

\section{Financiamiento}

El autor no declara fuente de financiamiento para la realización de este artículo. 


\section{Sobre el autor}

Edgar Alberto Rodríguez-Sánchez es profesional en Ciencias Militares de la Escuela Militar de Cadetes "General José María Córdova". Especialista en Derecho Administrativo y Constitucional, Seguridad y Defensa Nacional de la Escuela Superior de Guerra. Especialista en Alta Gerencia de la Universidad Militar Nueva Granada. Especialista en Docencia Universitaria de la Pontificia Universidad Javeriana y con estudios de Antropología y Familia en convenio de la Universidad Militar Nueva Granada y la Universidad Pontificia Ateneo de la Santa Cruz (Italia-Roma)

https://orcid.org/0000-0002-7622-7442 - Contacto: edgar.rodriguezsa@buzonejercito.mil.co

\section{Referencias}

Cardona Angarita, J. M. (2020). La doctrina militar colombiana en las prácticas de los oficiales durante el conflicto armado interno (1995-1998). Revista Cientifica General José María Córdova, 18(31), 607-625. https://doi.org/10.21830/19006586.606

Chandler, A. (2003). Strategy and structure: chapters in the history of the American Industrial Enterprise. Beardbooks.

Ciro, A., \& Correa, M. (2014). Transformación estructural del Ejército colombiano. Construcción de escenarios futuros. Revista Científica General José María Córdova, 12(13), 19-88. http://www.scielo.org.co/ pdf/recig/v12n13/v12n13a02.pdf

Consejo Nacional de Acreditación [CNA]. (2020). Glosario. https://www.cna.gov.co/1741/article-187835.html Dancy, J. (1993). Introducción a la epistemología contemporánea. Tecnos.

Ejército Nacional de Colombia. (2015). Plan Minerva. Proyecto Educativo Institucional. Publicaciones del Ejército Nacional.

Ejército Nacional de Colombia. (2016). MFE 1-01 Doctrina. Publicaciones del Ejército Nacional.

Ejército Nacional de Colombia. (2017a). MFE 5-0 Proceso de operaciones. Publicaciones del Ejército Nacional.

Ejército Nacional de Colombia. (2017b). Reglamento EJC 1-01. Doctrina y publicaciones militares del Ejército Nacional. Publicaciones del Ejército Nacional.

Ejército Nacional de Colombia. (2019). Manual MTE 5-0.1 Arte y diseño operacional. Publicaciones del Ejército Nacional.

Escuela Militar de Cadetes "General José María Córdova". (2020). Proyecto educativo del programa "Profesional en Ciencias Militares". ESMIC.

Ministerio de Defensa Nacional. (2010). Lineamientos curriculares Fuerzas Militares. Imprenta de las Fuerzas Militares.

Ministerio de Defensa Nacional. (2014). Proyecto Educativo de las Fuerzas Armadas PEFA. Imprenta de las Fuerzas Militares.

Ministerio de Defensa Nacional. (2019). Política Educativa de la Fuerza Pública (PEFuP) 2019-2032: hacia una educación inteligente, estratégica y de calidad. Ministerio de Defensa.

Najmanovich, D. (2008). Epistemología para principiantes. Longseller.

Ryabchuk, V. D. (2001). A new vision of military science. Military Thought, 10(6), 35-45. 